\title{
Organic thin film transistors and polymer light-emitting diodes patterned by polymer inking and stamping
}

\author{
Dawen Li and L Jay Guo ${ }^{1}$ \\ Department of Electrical Engineering and Computer Science, University of Michigan, Ann Arbor, \\ MI 48109-2122, USA \\ E-mail: guo@umich.edu
}

Received 7 February 2008, in final form 4 April 2008

Published 1 May 2008

Online at stacks.iop.org/JPhysD/41/105115

\begin{abstract}
To fully realize the advantages of organic flexible electronics, patterning is very important. In this paper we show that a purely additive patterning technique, termed polymer inking and stamping, can be used to pattern conductive polymer PEDOT and fabricate sub-micron channel length organic thin film transistors. In addition, we applied the technique to transfer a stack of metal/conjugated polymer in one step and fabricated working polymer light-emitting devices. Based on the polymer inking and stamping technique, a roll-to-roll printing for high throughput fabrication has been demonstrated. We investigated and explained the mechanism of this process based on the interfacial energy consideration and by using the finite element analysis. This technique can be further extended to transfer more complex stacked layer structures, which may benefit the research on patterning on flexible substrates.
\end{abstract}

(Some figures in this article are in colour only in the electronic version)

\section{Introduction}

In organic electronics, developing a suitable patterning technology for conducting polymer is of critical importance in realizing full-polymer, low-cost and high throughput processing on flexible substrates. Good patterning techniques for conducting polymers should meet several requirements: simple, high throughput, capable of small feature size and not compromise the conductive properties of the organic materials. Among the various techniques that have been investigated in the past, screen printing is a relatively simple patterning method, but it has difficulty in achieving resolutions better than $100 \mu \mathrm{m}$ [1], which is not suitable for defining the gate or channel length of the organic thin film transistors (OTFTs). Inkjet printing is widely used for organic electronics for feature sizes greater than $20 \mu \mathrm{m}$ [2]. It can also be used to create feature size down to $5 \mu \mathrm{m}$ [3], but would require highresolution pre-patterning which involves defining separate wetting (hydrophilic) and de-wetting (hydrophobic) regions. Inkjet printing also has rather low throughput because of its serial patterning scheme. Nanoimprint lithography (NIL)

\footnotetext{
${ }^{1}$ Author to whom any correspondence should be addressed.
}

has the capability to pattern polymers with nanometer scale resolutions [4], but requires an additional oxygen plasma etching step for removing residual layer in the imprinted region [5], which may damage functional materials such as conductive polymers.

Direct stamping of functional materials onto target substrates shows a great promise for low-cost, large area flexible electronics. Since direct pattern transfer is a purely additive process without any wet or dry etching involved, the functionality of organic materials can be well preserved. By using polydimethylsiloxane (PDMS) elastomer stamps the direct pattern transfer of functional materials can be easily achieved on a flexible substrate because the flexibility of a PDMS stamp can ensure a good conformal contact with flexible substrates. The material transferring by stamping technique has been applied to pattern metal contacts [6-9] and to transfer polymer thin films onto patterned substrates [10]. We recently developed the polymer inking and stamping technique [11] to directly pattern conducting polymer polyethylenedioxythiophene, doped with polystyrene sulfonic acid (PEDOT/PSS, Baytron AG high conductivity formulation) as source/drain contacts of micron-scale OTFTs. 

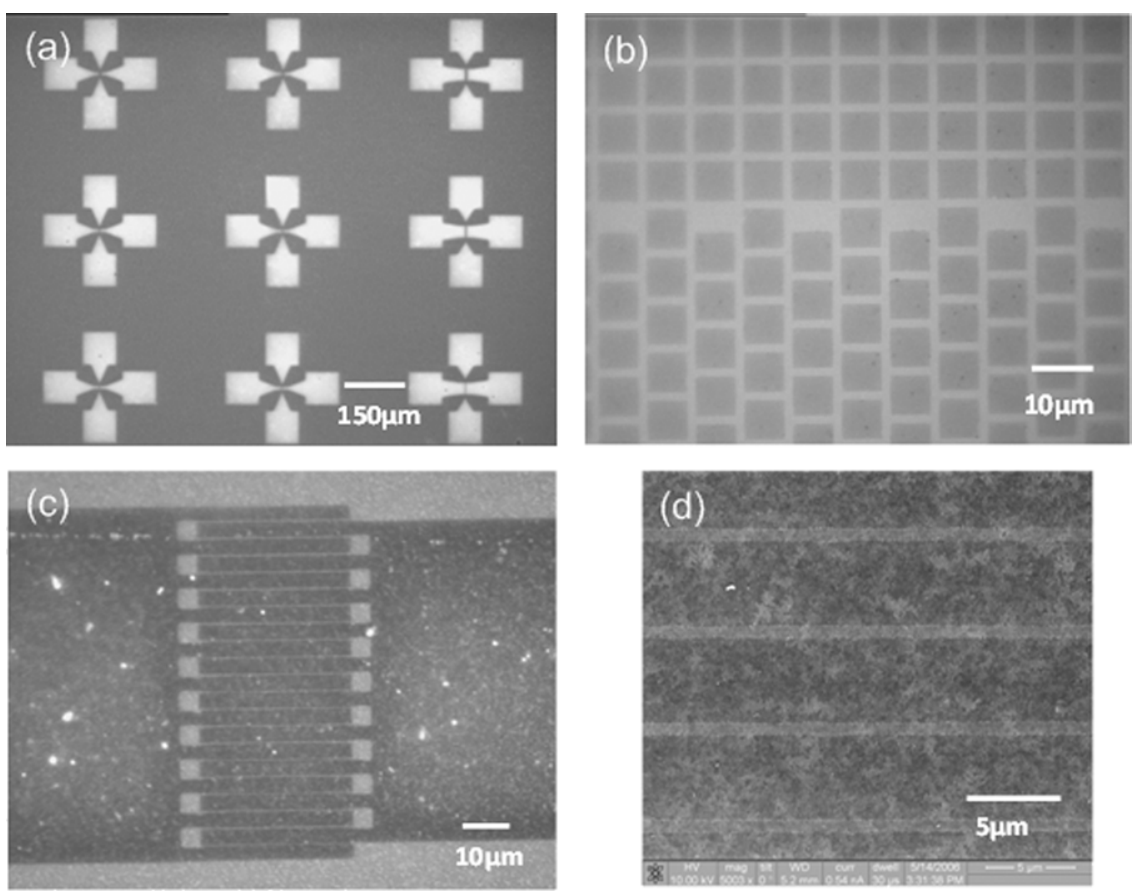

Figure 1. Optical micrographs of transferred PEDOT patterns on $(a) \mathrm{Si} / \mathrm{SiO}_{2}$ substrate with channel length from 2 to $10 \mu \mathrm{m}$, and $(b)$ on PES flexible substrate; $(c)$ interdigited sub-micron PEDOT pattern on $\mathrm{Si} / \mathrm{SiO}_{2}$ substrate with channel length of $0.6 \mu \mathrm{m}$ and $(d)$ scanning electron microscope (SEM) image of the PEDOT patterns with $0.6 \mu \mathrm{m}$ gap.

In this study, we investigate the pattern transfer mechanism in the inking and stamping process, and demonstrate sub-micron channel length OTFTs with interdigited PEDOT contacts. Moreover, we show that the process can be adapted to a rollto-roll printing process and achieved a minimum feature size of $5 \mu \mathrm{m}$. Furthermore, rather than only patterning single PEDOT layer, we demonstrated that the technique can be used to transfer a multi-layer polymer light-emitting diode (PLED) stack in one step.

\section{Patterning of sub-micron OTFTs by polymer inking and stamping}

As the name implies, the inking and stamping technique we developed here involves both inking and stamping steps. For the OTFT patterning, conducting polymer PEDOT is first 'inked' onto a PDMS stamp by spin-coating. PDMS stamps with source/drain patterns are made by casting and curing PDMS pre-polymer against a silicon master mould. In a subsequent 'stamping' step, the PEDOT coated stamp is placed on a substrate for making bottom contact (BC) OTFTs or on top of an organic semiconductor thin film for top contact (TC) OTFTs. Without external force, the PEDOT layer on top of the stamp protrusions is in conformal contact with the substrate due to the flexibility of the PDMS. After the PDMS stamp is removed, the PEDOT patterns are left on the substrate having identical dimensions as the source/drain patterns on the stamp. Figures 1(a) and $(b)$ show the transferred multiple micro-scale PEDOT patterns on a $\mathrm{Si} / \mathrm{SiO}_{2}$ substrate and a poly ether sulfone (PES) flexible substrate, respectively. Figures $1(c)$ and $(d)$ show sub-micron interdigited PEDOT source/drain patterns, which demonstrate that the polymer inking and stamping technique is capable of patterning OTFTs with sub-micron channel length.

The role of PDMS stamp used in the polymer inking and stamping pattern transfer is analogous to that of the photomask in photolithography. The process of sub-micron silicon master mould fabrication starts with a $\mathrm{Cr}$ deposition on a Si substrate with a $2 \mu \mathrm{m}$ thick oxide layer. Then spin-coated photo-resist is patterned by projection stepper lithography (GCA AS200 auto stepper) for sub-micron feature sizes. The $\mathrm{Cr}$ is wet etched in $\mathrm{Cr}$ etchant through the opened resist windows. A CHF3 plasma reactive ion etch (RIE) is carried out to transfer resist/Cr patterns into the oxide. Photo-resist and $\mathrm{Cr}$ layer together serve as RIE dry etching mask to prevent the sputtering of exposed $\mathrm{Cr}$ material. After RIE dry etching, photo-resist is removed by Pirahna cleaning [12], followed by $\mathrm{Cr}$ stripping in a $\mathrm{Cr}$ etchant. The fabricated silicon mould was further vapourcoated with $1 \mathrm{H}, 1 \mathrm{H}, 2 \mathrm{H}, 2 \mathrm{H}$-perfluorodecyltrichlorosilane $\left(130{ }^{\circ} \mathrm{C}\right.$ on hotplate for $\left.10 \mathrm{~min}\right)$ to achieve easy mould release after PDMS casting and curing.

Typical commercial PDMS (Sylgard 184, Dow Corning) is not suitable for producing sub-micron structures due to its low modulus, either causing pattern collapse or rounded corners [13]. Here we used a recently developed fast thermally curable and high-modulus PDMS [14] to make composite PDMS stamps [15] with sub-micron feature size. PDMS materials with high elastic modulus prevent closely spaced, sub-micron structures from sticking together. The process of making a composite PDMS stamp includes two steps. First, the liquid PDMS pre-polymer as described in [14] is poured onto the silicon master mould. After pumping out the bubbles in a vacuum oven (20-25 mTorr), the pre-polymer is cured at a temperature of $80^{\circ} \mathrm{C}$ for $10 \mathrm{~min}$, forming a thin 

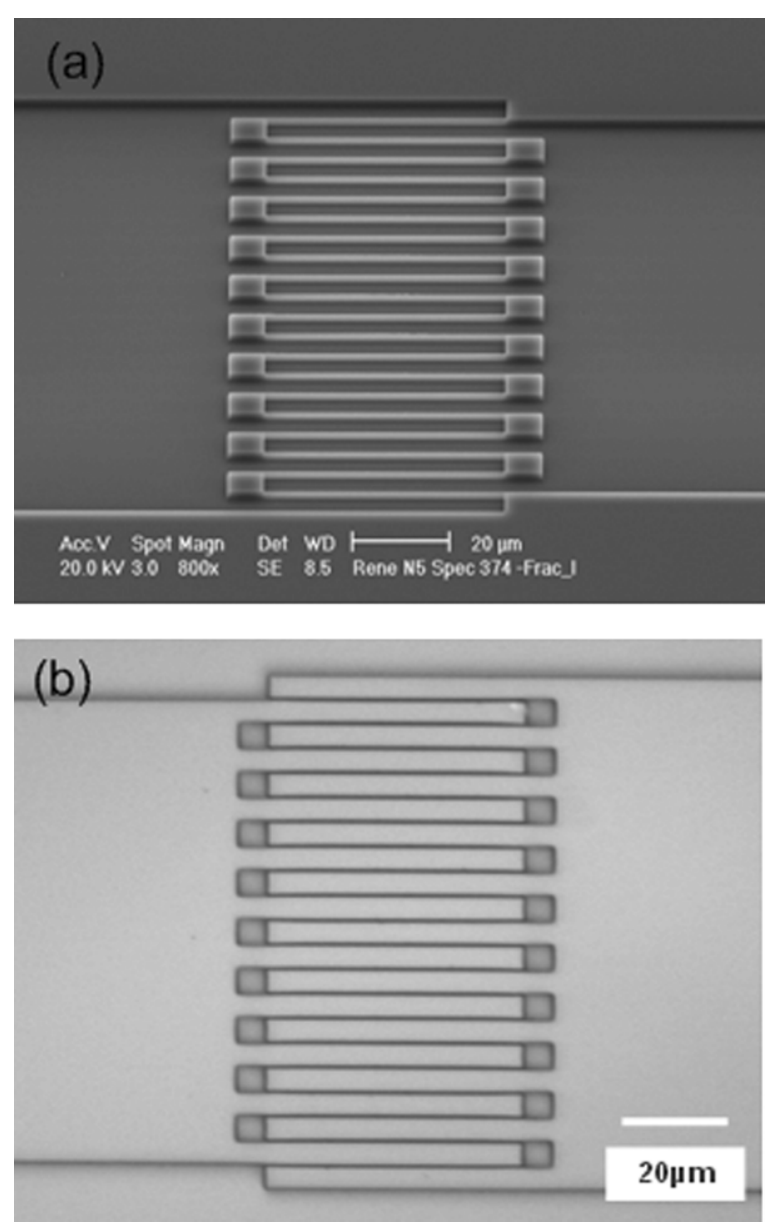

Figure 2. (a) SEM micrographs of silicon master mould with interdigited finger pattern $(0.8 \mu \mathrm{m}$ channel gap $)$, the sample is tilted $45^{\circ}$ degree for clear illustration of feature height of $2 \mu \mathrm{m}(b)$ optical micrographs of PDMS stamp cast from silicon master mould.

PDMS layer with precisely defined sub-micron features. Then conventional PDMS (Sylgard 184, Dow Corning) pre-polymer with base/curing agent loading ratio of $7: 1$ in weight is cast onto high-modulus PDMS layer. The cured PDMS $\left(80^{\circ} \mathrm{C}, 1 \mathrm{~h}\right)$ serves as a flexible supporting substrate for the whole PDMS stamp.

Silicon master mould with sub-micron interdigited electrode patterns and the replicated PDMS stamp are illustrated in figures $2(a)$ and $(b)$, respectively. The gaps between drain and source electrodes serve as the sub-micron channels.

\section{Mechanism of the polymer inking and stamping process}

In the polymer inking and stamping technique, the successful pattern transfer depends on the breaking of the inked layer on the PDMS stamp as well as the difference in adhesion energy at various interfaces. An atomic force microscopy (AFM) image [11] of the cross-sectional profile of the spincoated PEDOT on the PDMS stamp reveals that the PEDOT thin film at the very edge of the stamp protrusions is thinner than on the other surfaces of the PDMS stamp, and therefore it is the weakest point when the PDMS stamp is removed from the substrate. We performed finite element simulation with ANSYS, where the PEDOT film was modelled as a linear isotropic material with Young's modulus of $2 \mathrm{GPa}$, and Poisson's ratio of 0.44 [16]. As shown in figure 3, when a pulloff force is applied to the PEDOT film as the stamp is separated from the substrate, maximum stress occurs at the edge of the PDMS protrusion. When the maximum stress exceeds the yield stress of the PEDOT material, the PEDOT thin film will break first around the protrusion edges.

Once the PEDOT thin film breaks at the edge of the protrusion patterns, the PEDOT patterns can be transferred successfully onto a substrate if the adhesion between the PEDOT and the substrate is stronger than that between the PDMS and the PEDOT. For the PEDOT pattern transfer directly onto a substrate without an organic active layer (e.g. an oxide surface), due to the addition of a coupling agent Silquest 187 (Dow Corning Co.) in the PEDOT aquatic solution, and oxygen plasma treatment of the substrate, the adhesion force between the PEDOT and the substrate is always larger than that between the PEDOT and the PDMS stamp. As a result, the PEDOT pattern transfer always succeeds. For the PEDOT pattern transfer onto a substrate with an organic active layer (e.g. pentacence), a stronger adhesion of the organic active layer onto the substrate is required, otherwise the PDMS stamp with the PEDOT film will peel off the organic material from the substrate, instead of transferring the PEDOT pattern onto the organic layer. In this study, a successful PEDOT pattern transfer onto the pentacene thin film relies on strong adhesions at both PEDOT/pentacene and pentacene/substrate interfaces, whereas the weakest adhesion should exist at the PDMS/PEDOT interface to ensure that the PDMS/PEDOT separation takes place first at this interface. Adhesion between pentacene thin film and oxide substrate can be enhanced by the octadecyltrichlorosilane (OTS) treatment of the oxide surface. The OTS treatment also improves the electrical performance of the pentacene OTFTs because of the improved ordering of the pentacene molecules [17-19].

To quantify the adhesion forces at the various interfaces, we performed contact angle measurement with water and ethylene glycol as the test liquids. The contact angles were measured by using a goniometer (KSV Instruments Ltd, CAM100). Adhesion forces (work of adhesion) at different interfaces can be calculated from the measured contact angles by using the procedure described in [20]. The calculated adhesion forces at different interfaces are summarized in table 1.

The work of adhesion at the interface between the pentacene and the oxide with OTS treatment is $46.7 \mathrm{~mJ} \mathrm{~m}^{-2}$, while that between the pentacene and the oxide without OTS treatment is $42.9 \mathrm{~mJ} \mathrm{~m}^{-2}$, which supports the experimental observation that OTS treatment of oxide improves the adhesion of pentacene on oxide, which should improve the PEDOT pattern transfer fidelity. The work of adhesion at the interface between the pentacene and PEDOT is $35.8 \mathrm{~mJ} \mathrm{~m}^{-2}$, which is less than the work of adhesion between the pentacene and the oxide substrate. Thus, in principle the PDMS stamp should not stick off the pentacene from the oxide substrate. 
(a)

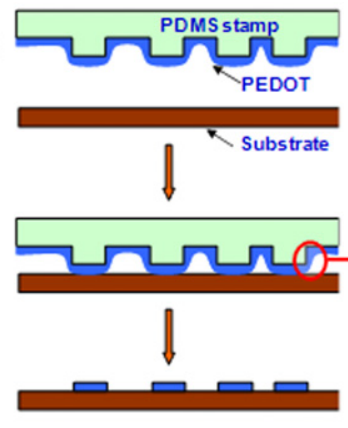

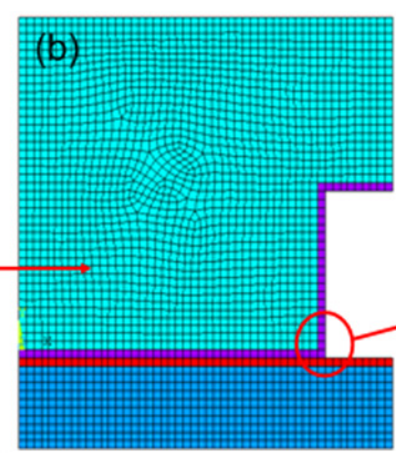

Figure 3. (a) Schematic of PEDOT inking and stamping, $(b)$ modelling of the finite element simulation, PEDOT thin film thickness of $100 \mathrm{~nm}(c)$ colour map of simulated stress distribution in the PEDOT layer during stamp removal. Maximum stress in the PEDOT thin film occurs at the corner of the PDMS stamp protrusion when PDMS stamp is removed from the substrate.

Table 1. The calculated work of adhesion $\left(\mathrm{mJ} \mathrm{m}^{-2}\right)$ at the different interfaces.

\begin{tabular}{|c|c|}
\hline Interfaces & $\begin{array}{l}\text { Work of adhesion } \\
\left(\mathrm{mJ} \mathrm{m}^{-2}\right)\end{array}$ \\
\hline Pentacene $/ \mathrm{SiO}_{2}$ with OTS treatment & 46.7 \\
\hline Pentacene $/ \mathrm{SiO}_{2}$ without OTS treatment & 42.9 \\
\hline PEDOT/Pentacene & 35.8 \\
\hline PDMS (10 $\mathrm{s} \mathrm{O}_{2}$ plasma treatment)/PEDOT & 151.3 \\
\hline PDMS (no $\mathrm{O}_{2}$ plasma treatment)/PEDOT & 9.4 \\
\hline
\end{tabular}

However, in reality some parts of pentacene thin film are peeled off from the substrate, which may be due to insufficient difference of work of adhesion between these two interfaces, or complex stress distribution when removing the PDMS stamp. Before spin-coating PEDOT onto the PDMS stamp, a brief $\mathrm{O}_{2}$ plasma treatment of the PDMS stamp is necessary to ensure uniform coating of the PEDOT. The work of adhesion at the interface between the PEDOT and the PDMS stamp treated with $10 \mathrm{~s} \mathrm{O}_{2}$ plasma treatment is $151.3 \mathrm{~mJ} \mathrm{~m}^{-2}$, while only $9.4 \mathrm{~mJ} \mathrm{~m}^{-2}$ for without $\mathrm{O}_{2}$ plasma treatment. This large difference explains that it is very necessary to recover the hydrophobility of the PDMS stamp by baking the entire assembly before removing the PDMS stamp [11]. Only when the adhesion energy between the PEDOT and the PDMS stamp is reduced to less than that at the pentacene and the PEDOT interface $\left(35.8 \mathrm{~mJ} \mathrm{~m}^{-2}\right)$, successful PEDOT pattern transfer onto pentacene film could occur. Such an analysis could provide a guideline to apply the polymer inking and stamping technique to other pattern transfer processes.

\section{Extending to a roll-to-roll printing process}

The roll-to-roll printing method is very attractive for organic electronics because of its high-throughput and low-cost fabrication, and a continuous room-temperature processing over large-area flexible substrates. Based on the polymer inking and stamping technique, roll-to-roll printing of conducting polymer on a flexible substrate has been demonstrated by using a roller-printing machine. The PDMS stamp with spin-coated PEDOT is attached to one roller. The flexible substrate is fed in on the other roller. As both rollers rotate, the PDMS stamp contacts flexible substrate,

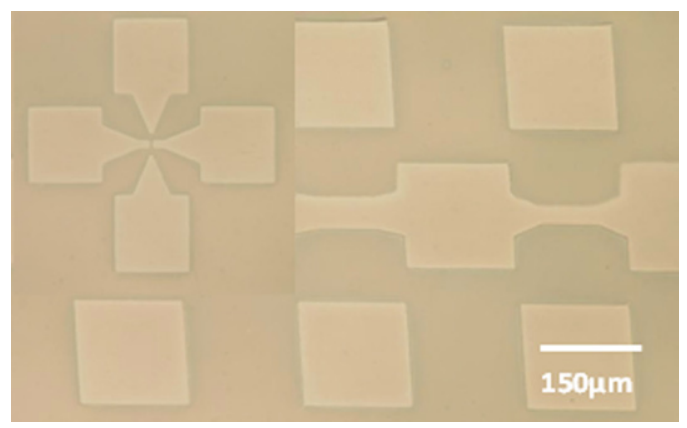

Figure 4. Optical micrograph of the conducting polymer PEDOT pattern on PET flexible substrate by using a roll-to-roll printing machine. The inset shows the transferred PEDOT pattern with $5 \mu \mathrm{m}$ channel gap.

transferring PEDOT patterns onto the flexible substrate. Figure 4 shows the printed PEDOT patterns on poly(ethylene terephthalate) (PET) flexible substrate with a printing speed of $12 \mathrm{~mm} \mathrm{~s}^{-1}$. A minimum feature size of $5 \mu \mathrm{m}$ has been achieved. Such roll-to-roll patterning technique could be applied to the fabrication of full-polymer organic devices on flexible substrates, especially patterning OTFT backplane and organic polymer LEDs for active-matrix OLED flexible displays [21].

\section{Multi-layer PLED patterning in one step}

In addition to the single layer PEDOT transfer for patterning the electrodes of OTFTs, we further demonstrate that the inking and stamping technique is capable of patterning multilayer PLED structure in one step. A schematic of 3-layer inking and stamping for PLEDs is illustrated in figure 5. Three layers of PLEDs are 'inked' onto the PDMS stamp in sequence. A $30 \mathrm{~nm}$ Au thin film followed by $30 \mathrm{~nm} \mathrm{Al}$ cathode layer is first deposited onto a PDMS stamp by e-beam evaporation (chamber vacuum of $2 \times 10^{-6}$ Torr, deposition rate of $2 \AA \mathrm{s}^{-1}$ ). Before Au/Al deposition, the PDMS stamp is briefly treated with $\mathrm{O}_{2}$ plasma $(250 \mathrm{mTorr}, 80 \mathrm{~W}, 10 \mathrm{~s})$ to provide a good adhesion between the deposited Au and the PDMS. Following the metal deposition, poly[2-methoxy5-(2'-ethyl-hexyloxy)-1,4-phenylene vinylene] (MEH-PPV) light-emitting polymer (ADS 133YE) and PEDOT hole 
transport material (Baytron VP CH 8000, OLED grade) are subsequently spin-coated onto the $\mathrm{Au} / \mathrm{Al}$ coated PDMS stamp at $3 \mathrm{krpm}$ for $30 \mathrm{~s}$. MEH-PPV polymer is diluted in toluene (2\% in weight), and filtered with a $0.7 \mu \mathrm{m}$ filter. By stamping onto a substrate, the 3 layers of PLED devices are transferred onto the substrate in one step. The photograph in figure $6(a)$ demonstrates high-yield multi-layer PLED pattern transfer on the flexible substrate based on the polymer inking and stamping technique. The optical micrograph in figure $6(b)$ shows the top view of the clean multi-layer PLED patterns on an indium-tinoxide (ITO)-coated PET flexible substrate. The cross-section view of the transferred 3-layer structure is shown in figure $6(c)$. In this SEM image, the $\mathrm{Au} / \mathrm{Al}(60 \mathrm{~nm})$ cathode layer, the MEHPPV (90 nm) light-emitting layer and the PEDOT $(90 \mathrm{~nm})$ hole transport layer can be clearly identified.

The adhesion force at different interfaces again plays a critical role in the successful 3-layer pattern transfer. The largest interface adhesion is desired to occur at the PEDOT/ITO interface, while the weakest adhesion should

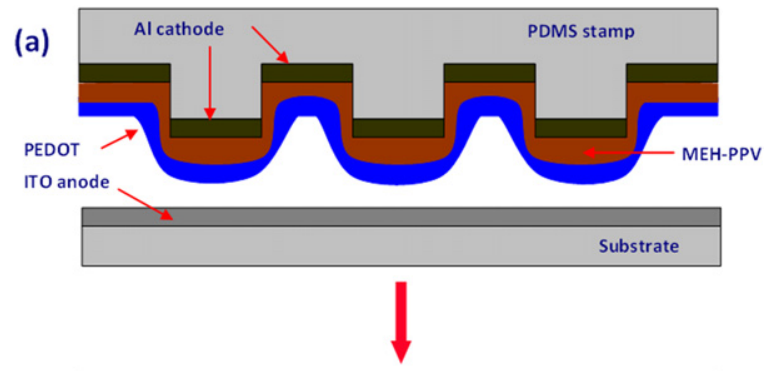

(b)
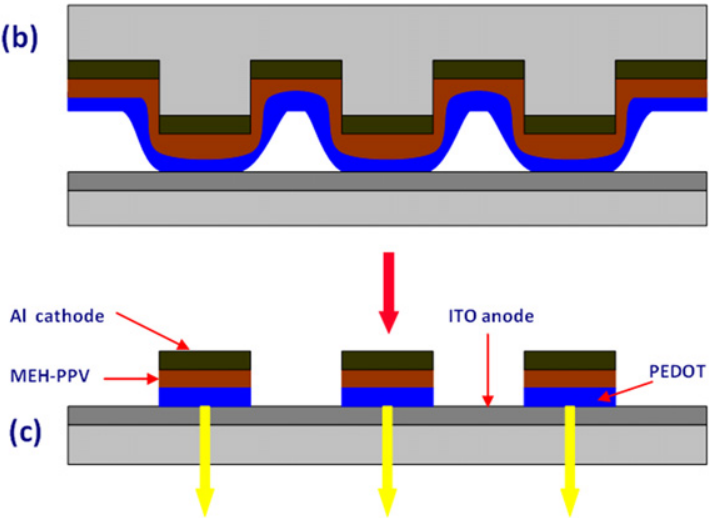

Figure 5. Schematic of the inking and stamping process for patterning multi-layer PLEDs in one step, (a) 3-layer inking on the PDMS stamp, $(b)$ stamping onto a substrate and $(c)$ transferred 3-layer stack patterns. be at the PDMS/metal interface. The adhesion force of the other two interfaces should be in between these two to ensure a successful separation occurs at the PDMS/metal interface. Similarly to the polymer inking and stamping for transferring PEDOT patterns, glycerol is added into PEDOT to prevent the complete dry-out after spin-coating the PEDOT; and adhesion coupling agent Siliquest 187 is added into PEDOT to increase the adhesion at the PEDOT/ITO interface. The whole assembly is baked at $80^{\circ} \mathrm{C}$ for $2 \mathrm{~min}$ to reduce the adhesion at the PDMS/metal interface. Prior to spin-coating of light-emitting polymer MEH-PPV, polymer adhesion promoter is coated to form a monolayer on the Al surface, which enhances the adhesion between $\mathrm{Al}$ and MEH-PPV. Also, after coating of MEH-PPV, a brief $\mathrm{O}_{2}$ plasma treatment ( $250 \mathrm{mTorr}, 80 \mathrm{~W}, 20 \mathrm{~s}$ ) is necessary to increase the MEH-PPV surface energy, so that hole transport layer PEDOT could be spin-coated uniformly onto the MEH-PPV surface.

\section{Characterization of patterned OTFTs and PLEDs}

Figures 7(a) and (b) show the electrical output and transfer characteristics of TC pentacene TFTs with interdigited PEDOT electrodes, respectively. Pentacene thin film of $30 \mathrm{~nm}$ was thermally deposited onto OTS-treated oxide substrates in vacuum $\left(5 \times 10^{-6}\right.$ Torr $)$ and with a deposition rate of $0.5 \AA \mathrm{s}^{-1}$. To obtain standard electrical characteristics for submicron OTFTs, oxide thickness of $45 \mathrm{~nm}$ is used to ensure long channel behaviour (i.e. transverse electric field $E_{x} \gg$ longitudinal field $E_{y}$ along the channel), avoiding the inability to reach drain current saturation which is widely observed in short-channel OTFT devices [22-26]. To characterize the TC OTFT devices, metal gallium-indium liquids are used as a probe cushion between the PEDOT contact and a metal probe for reliable measurements. This is because the conducting polymer PEDOT electrode layer can be easily punctured by a sharp probe.

A maximum drain current in saturation regime $\left(V_{\mathrm{DS}}=\right.$ $\left.V_{\mathrm{GS}}=-5 \mathrm{~V}\right)$ of $\sim 40 \mu \mathrm{A}$ has been achieved for $1 \mu \mathrm{m}$ wide channel OTFTs. The drain currents of the OTFTs with interdigited PEDOT contacts are significantly improved due to extremely large geometric ratio $W / L$ [27]. The field-effect mobility has been extracted in the saturation regime based on the following equation, where $C_{\text {in }}$ is the insulator capacitance, and $V_{\mathrm{T}}$ is the threshold voltage.

$$
I=\frac{W}{2 L} \mu C_{\mathrm{in}}\left(V_{\mathrm{GS}}-V_{\mathrm{T}}\right)^{2} .
$$
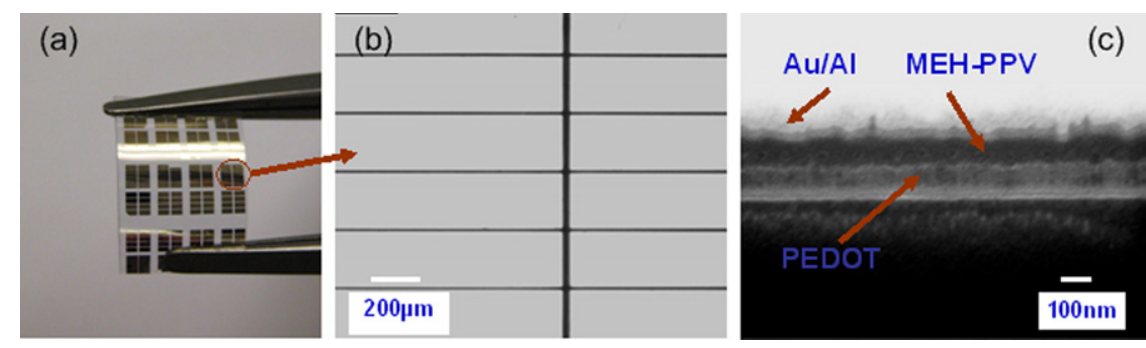

Figure 6. (a) Photograph of the transferred multi-layer PLEDs on the PET flexible substrate, $(b)$ top view of the multi-layer PLED patterns, (c) cross-sectional SEM image of the transferred 3 layers, $\mathrm{Au}(30 \mathrm{~nm}) / \mathrm{Al}(30 \mathrm{~nm}) / \mathrm{MEH}-\mathrm{PPV}(90 \mathrm{~nm}) / \mathrm{PEDOT}(90 \mathrm{~nm})$ from top to bottom. 

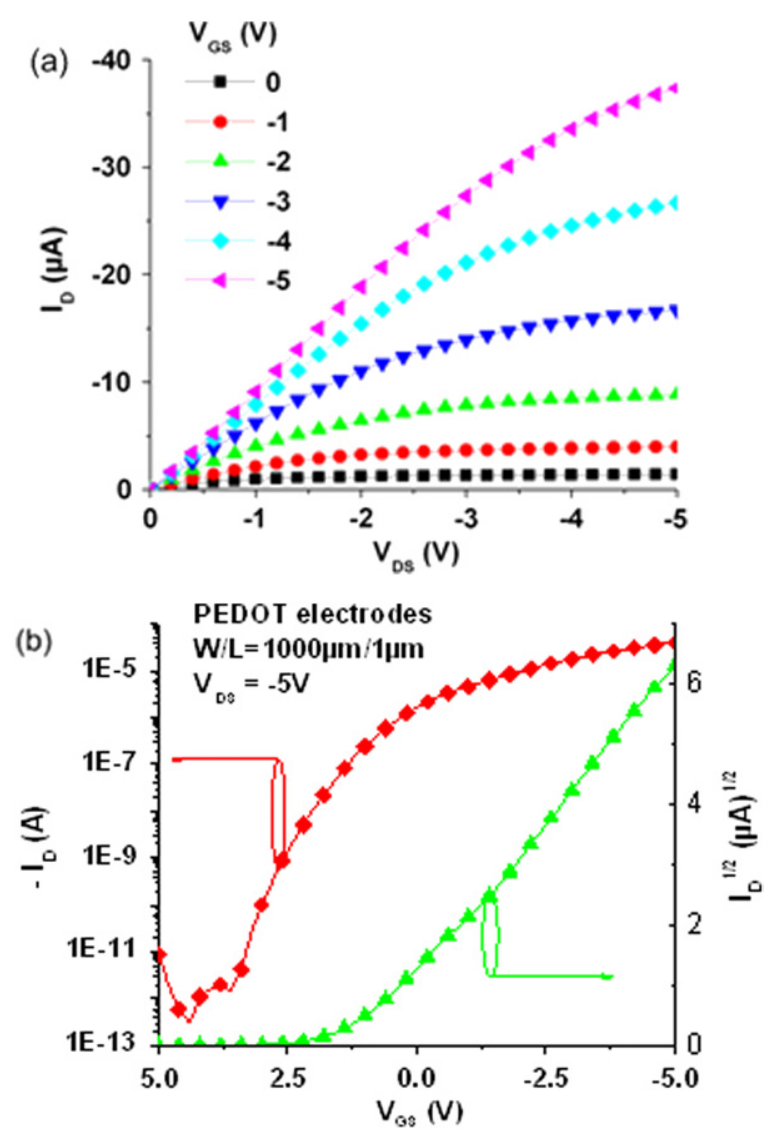

Figure 7. (a) Electrical output characteristics and $(b)$ transfer characteristics of the TC pentacene OTFTs

$(W / L=1000 \mu \mathrm{m} / 1.0 \mu \mathrm{m})$ with interdigited PEDOT electrodes.

The extracted field-effect mobility of $3.3 \times$ $10^{-2} \mathrm{~cm}^{2} \mathrm{~V}^{-1} \mathrm{~s}^{-1}$, threshold voltage of $1.2 \mathrm{~V}$, subthreshold swing of $0.5 \mathrm{~V} /$ decade, very low OFF current of $10^{-12} \mathrm{~A}$, and high ON/OFF current ratio of $10^{8}$ are obtained. Note that the extracted mobility from pentacene TC OTFTs with PEDOT interdigited finger configuration is about one order of magnitude lower than that from micron-scale pentacene TC OTFTs with typical PEDOT electrodes. This lower mobility can be attributed to two reasons. One reason is that the extracted mobility is reduced for shorter channel length because the effect of contact resistance becomes more significant [11]. Another reason may come from the voltage drop on the PEDOT interdigited finger electrodes. The PEDOT finger has length of $50 \mu \mathrm{m}$, a width of $5 \mu \mathrm{m}$, and a thickness of about $100 \mathrm{~nm}$. The measured sheet resistance of high conductivity PEDOT thin film is about $2400 \Omega / \square$. Thus, the resistance of each finger is around $24 \mathrm{k} \Omega$. The maximum voltage drop with a drain current level of $40 \mu \mathrm{A}$ is about $0.96 \mathrm{~V}$, which is a significant fraction of the applied drain voltage of $5 \mathrm{~V}$. The sheet resistance of the transferred PEDOT thin film can be reduced by increasing its thickness, which can be achieved by using a lower spin-speed during spin-coating of PEDOT. However, a trade-off exists because a low spin-speed could result in non-uniformity of the coated PEDOT film. We used a spin-speed of $3 \mathrm{krpm}$ here to ensure the uniformity of the PEDOT thin film.

The fabricated PLED device was characterized by using the semiconductor parameter analyzer (HP 4145) with the

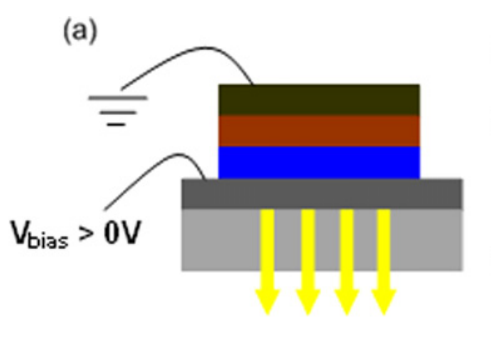

Al cathode

MEH-PPV

PED OT (HTL)

ITO anode

PET substrate

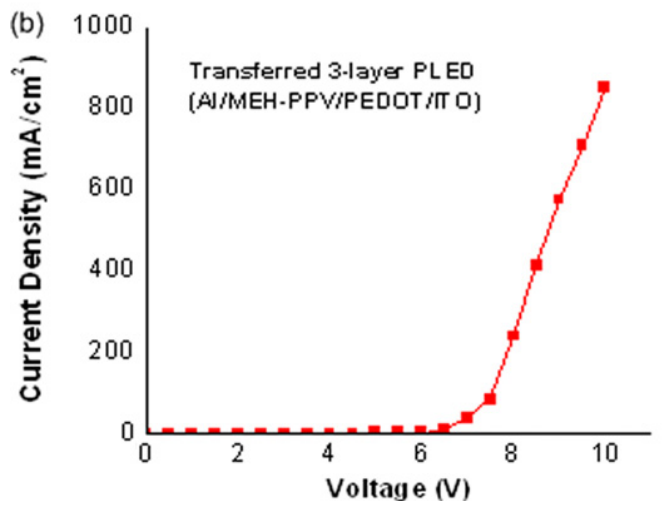

Figure 8. (a) Schematic of characterization and (b) $J-V$ characteristics of the PLEDs (Al/MEH-PPV/PEDOT/ITO) patterned by the 3-layer inking and stamping.

Al layer as cathode, and transparent ITO as anode shown in figure $8(a)$. Before characterization the patterned multilayer PLEDs are annealed at $130^{\circ} \mathrm{C}$ on a hotplate for $9 \mathrm{~h}$ to enhance the interface adhesion between the emissive MEH-PPV polymer and the $\mathrm{Al}$ cathode, which improves the efficiency of PLEDs [28]. Figure $8(b)$ shows the PLED characteristics of current density versus applied bias. The electrical characteristics of PLEDs made by multi-layer pattern transfer are similar to those standard-fabricated PLEDs with the same organic materials and electrodes [29]. The turn-on voltage of PLED is about $7 \mathrm{~V}$, higher than that reported in [29] (around $6 \mathrm{~V}$ ), and the yellow light emits from the bottom of the transparent ITO substrate.

\section{Conclusions}

In summary, the sub-micron pentacene OTFTs with the interdigited PEDOT electrodes were patterned by the polymer inking and stamping technique. The driving currents of the pentacene OTFTs with the interdigited PEDOT contacts are significantly increased due to the high channel width-to-length ratio. The mechanism of the inking and stamping pattern transfer was systematically investigated with the finite element simulation and the interface adhesion energy calculation. Based on the polymer inking and stamping process, the roll-to-roll printing of the conducting polymer PEDOT on the flexible substrate has been demonstrated. Finally the inking and stamping pattern transfer technique is not only capable of patterning single PEDOT layer for OTFTs but also demonstrates for transferring a multi-layer PLED structure in one step. In principle, this technique can be extended to transfer more complex stacked layer structures. 


\section{Acknowledgments}

The authors would like to thank Ms Yiying Zhao for pentacene deposition. This work was supported by the NSF grant CMII 0700718 and NSFC grant No 60528003.

\section{References}

[1] Min G 2003 Synth. Met. 135-136 141-3

[2] Kipphan H 2001 Handbook of Print Media: Technologies and Production Methods (New York: Springer)

[3] Sirringhaus H, Kawase T, Friend R H, Shimoda T, Inbasekaran M, Wu W and Woo E P 2000 Science $2902123-6$

[4] Chou S Y, Krauss P R and Renstrom P J 1996 Science $27285-7$

[5] Guo L J 2004 J. Phys. D: Appl. Phys. 37 R123-41

[6] Hur S-H, Khang D-Y, Kocabas C and Rogers J A 2004 Appl. Phys. Lett. 85 5730-2

[7] Loo Y-L, Willett R L, Baldwin K W and Rogers J A 2002 Appl. Phys. Lett. 81562

[8] Wang Z, Yuan J, Zhang J, Xing R, Yan D and Han Y 2003 Adv. Mater. 15 1009-12

[9] Kim C, Shtein M and Forrest S R 2002 Appl. Phys. Lett. 80 4051-3

[10] Tan L, Kong Y P, Bao L-R, Huang X D, Guo L J, Pang S W and Yee A F 2003 J. Vac. Sci. Technol. B 21 2742-8

[11] Li D and Guo L J 2006 Appl. Phys. Lett. 88063513

[12] Tauber S W a R N 2000 Silicon processing for the VLSI era Process Technology vol 1 (Sunset Beach CA: Lattice Press)

[13] Hui C Y, Jagota A, Lin Y Y and Kramer E J 2002 Langmuir 18 1394-407
[14] C Pina-Hernandez J-S K, Guo L J and Fu P-F 2007 Adv. Mater. 19 1222-7

[15] Odom T W, Love J C, Wolfe D B, Paul K E and Whitesides G M 2002 Langmuir 18 5314-20

[16] Lang U and Dual J 2007 The Mechanical Behavior of Materials X Part 1: 10th Int. Conf. on the Mechanical Behaviour of Materials Key Eng. Mater. 345-346 1189-92

[17] Gundlach D J, Kuo C S, Sheraw C D, Nichols J A and Jackson T N 2001 Proc. SPIE-Int. Soc. Opt. Eng. 4466 54-64

[18] Song C-K, Koo B-W, Lee S-B and Kim D-H 2002 Japan. J. Appl. Phys. 41 2730-4

[19] Locklin J, Ling M M, Sung A, Roberts M E and Bao Z 2006 Adv. Mater. 18 2989-92

[20] Wang Z, Zhang J, Xing R, Yuan J, Yan D and Han Y 2003 J. Am. Chem. Soc. 125 15278-9

[21] Zhou L, Wanga A, Wu S-C, Sun J, Park S and Jackson T N 2006 Appl. Phys. Lett. 88083502

[22] Chabinyc M L, Lu J-P, Street R A, Wu Y, Liu P and Ong B S 2004 J. Appl. Phys. 96 2063-70

[23] Collet J, Tharaud O, Chapoton A and Vuillaume D 2000 Appl. Phys. Lett. 761941

[24] Xu Y and Berger P R 2004 J. Appl. Phys. 95 1497-501

[25] Leufgen M, Bass U, Muck T, Borzenko T, Schmidt G, Geurts J, Wagner V and Molenkamp L W 2004 Synth. Met. $146341-5$

[26] Torsi L, Dodabalapur A and Katz H E 1995 J. Appl. Phys. 78 1088-93

[27] Austin M D and Chou S Y 2002 Appl. Phys. Lett. 81 4431-3

[28] Lee T-W and Park O O 2000 Adv. Mater. 12 801-4

[29] Sohn1 S, Park1 K, Lee1 D, Jung1 D, Kim H M, Manna U, Yi J, Boo J-H, Chae H and Kim H 2006 Japan. J. Appl. Phys. 45 3733-6 